\title{
REGIONALISMO DELLA DIFFERENZIAZIONE E RIORDINO DELLE AUTONOMIE INFRAREGIONALI. UN GIOCO (ISTITUZIONALE) "NON A SOMMA ZERO"
}

di Giuseppe Carlo Ricciardi

\section{Crisi delle autonomie e autonomie nella crisi}

Nell'ultimo decennio il sistema delle autonomie di molti Stati nazionali aderenti all'Unione Europea ha conosciuto una stagione informata alla razionalizzazione. Si tratta di una reazione ai rilievi formulati da alcune Istituzioni europee ${ }^{1}$, che hanno ravvisato nella dimensione multilivello dei governi locali degli Stati membri maggiormente in difficoltà dal punto di vista finanziario una fonte di spesa da rivedere in chiave riduttiva $^{2}$, alla luce della crisi economica che ha colpito l'Europa ${ }^{3}$.

Dipartimento di Scienze Politiche e Sociali, Università di Pavia.

Questo saggio è una versione rivista e integrata dell'Audizione tenuta il 17 ottobre 2019 presso la Commissione Speciale "Autonomia e Riordino Autonomie Locali” del Consiglio regionale della Lombardia.

1 Si rammenta in questa sede solo la lettera della BCE all'Italia del 5 agosto 2011, in seguito accompagnata da una nota raccomandazione del Consiglio (COUNCIL - Recommendation 2014/C 247/11 on the National Reform Programme 2014 of Italy and delivering a Council opinion on the Stability Programme of Italy, 2014, 8 July 2014).

2 Cfr., per alcuni Paesi a tradizione amministrativa napoleonica come il nostro, il $\mathrm{Me}$ morando de Entendimento sobre as condicionalidades de Política Económica de 17 de maio de 2011, destinato al Portogallo, oppure, per quanto attiene alla Spagna, il Memorando de Entendimiento sobre condiciones de Politica Sectorial Financiera de 23 de julio de 2012, nonché l'Acuerdo Marco de Asistencia Financiera, de julio de 2012. Allo stesso modo, veniva rivolto alla Grecia il Memorandum of Understanding on Specific Economic Policy Conditionality of 9 February 2012, mentre si riferiva alla Francia l'Evaluation $d u$ programme national de réforme et du programme de stabilité 2013 du 29 mai 2013. Sul rapporto tra riforme territoriali ed istanze di derivazione europea cfr. E. ONGARO, L'influence de la nouvelle gouvernance européenne sur la réforme de l'administration publique dans les États de tradition administrative napoléonienne-Plaidoyer pour l'adoption d'un nouvel agenda de recherche, in "Revue française d'administration publique", n. 1, 2012.

$3 \mathrm{Ci}$ si riferisce alla crisi deflagrata con la fine degli anni Duemila, che si è velocemente 
Ne è conseguita in Italia una stagione di continui aggiustamenti normativi, caratterizzata dall'affastellarsi di provvedimenti statali di riordino alluvionali e, in certi casi, incoerenti tra loro, cui le Regioni hanno replicato attraverso leggi regionali di adeguamento. Tra le misure più incisive, una menzione particolare merita la riforma territoriale intervenuta con legge 56/2014, cui avrebbe dovuto far seguito un'ampia revisione della Costituzione, in seguito mai entrata in vigore a causa dell'esito negativo del referendum costituzionale svoltosi nel dicembre 2016. Molte sono state le voci critiche espresse sia sul merito, sia sul metodo adottato dai decisori politici nazionali; tra esse, alcune hanno sottolineato gli effetti determinati da siffatto uso congiunturale delle autonomie, che ha condotto all'attuale contesto disarticolato e poco coerente.

Quello italiano resta un sistema nel quale le Regioni faticano ad esercitare la propria potestà legislativa con risultati significativi, strette come sono nelle rigide maglie del riparto di competenze individuato dalle disposizioni del Titolo V della Costituzione4; un contesto nel quale persistono preoccupanti differenziali di performance - già denunciati decenni $\mathrm{fa}^{5}$ - tra Regioni in grado di esprimersi autorevolmente, dispiegando politiche pubbliche rivolte all'innovazione istituzionale e sociale e altre, invece, incapaci di creare valore ed adottare provvedimenti normativi e amministrativi degni di nota.

Anche il livello intermedio del governo territoriale sconta delle contraddizioni. A fronte dell'auspicata abolizione delle Province promossa dal Governo su impulso sovranazionale - queste ultime sono rimaste, infine, enti costituzionalmente necessari, pur essendo state medio tempore spogliate di prerogative ${ }^{6}$ che le rendevano il cen-

propagata in molti Paesi occidentali conducendo alla sperimentazione di politiche assai rigoristiche da parte delle istituzioni sovranazionali.

4 Specialmente per come interpretato da parte della Corte costituzionale.

5 Cfr. L. Paladin, Presentazione, in AA.VV., "La prima legislatura regionale 19701975”, Milano, Giuffrè, 1976.

6 Si pensi alla pianificazione strategica, riconosciuta alle Città metropolitane dalla legge 56/2014 (cfr. art. 1, comma 2 e art. 1, comma 44) ma solo in via surrogatoria alle Province (come desumibile dalla mancata previsione del riferimento allo sviluppo strategico nella norma di principio di cui all'art. 1, comma 3, che riappare solamente nell'art. 1, comma 86). Da un punto di vista sistematico, sul fronte dei principi, queste ultime potrebbero recuperare prerogative di sviluppo strategico dei territori anche sulla base del TUEL; tuttavia, essendo state spogliate di funzioni e risorse umane, tale competenza è andata disperdendosi e non è stata ripristinata al pari di quanto non avvenisse prima della riforma, come pure si sarebbe potuto auspicare accadesse. 
tro nevralgico di molte relazioni inter-istituzionali, anche sul fronte della capacità di corrispondere a esigenze di attrattività e competitività. Esse hanno condiviso con le neo-istituite Città metropolitane seppur secondo una scala più acuta di severità - $\mathrm{i}$ tagli lineari alle risorse umane e finanziarie che, rispettivamente, hanno impoverito le prime del loro più grande patrimonio, avente natura immateriale, ed hanno frenato le seconde, le quali dopo una gestazione durata venticinque anni ${ }^{7}$ sono state finalmente istituite a seguito della citata legge $56 / 2014$, salvo risultare immediatamente appesantite dalle istanze di revisione della spesa nella delicata fase di avviamento ${ }^{8}$.

Inoltre resta elevato il numero di Comuni, incrementalmente destinatari di sempre nuovi compiti ed adempimenti, rispetto ai quali è stato riconfermato il paradigma quasi paradossale dell'uniformità: uno statuto giuridico-amministrativo che - riconoscendo loro medesime funzioni amministrative ed identici adempimenti - vede disciplinati rigorosamente allo stesso modo Comuni di poche decine di abitanti e realtà istituzionali demograficamente imponenti, situate al centro delle più rilevanti relazioni internazionali.

Una vista di sistema riconsegna un quadro complessivo nel quale si giustappongono dinamiche tra loro antitetiche. Da un lato, si registrano tendenze spiccatamente centripete, che riecheggiano nella contrapposizione tra una diffusa debolezza del centro ${ }^{9}$, che pure aspira ad irrobustire il proprio ruolo - anche nel governo dei fenomeni eco-

7 Gli Enti metropolitani erano già stati previsti nel nostro ordinamento a partire dalla legge 142/1990, la quale prevedeva un regime speciale per le Aree metropolitane. Preso atto delle difficoltà attuative incontrate, la legge 265/1999, poi recepita negli artt. 22 ss. del D.lgs. 267/2000, aveva riordinato la disciplina volta all'istituzione delle Città metropolitane, successivamente riconosciute quali Enti costituzionalmente garantiti a seguito della riforma del Titolo V della parte seconda della Costituzione (art. 114 Cost.). Peraltro, dopo le previsioni ad esse dedicate nell'ambito della legge 131/2003 e della legge 42/2009 - ed a seguito del tentativo di istituzione promosso con decreto legge nel 2012, naufragato in conseguenza della nota sentenza Corte cost. 220/2013 - solo con la legge 56/2014 si è offerto l'impulso decisivo affinché le Città metropolitane conoscessero attuazione sul territorio nazionale.

8 Per una ricostruzione, cfr. F. Osculati, La dotazione organica e finanziaria degli Enti di area vasta. Un assetto ancora provvisorio ovvero una vicenda dirigistica, in G.C. RicCIARDI, A. VENTURI (a cura), "La riorganizzazione territoriale e funzionale dell'Area vasta", Torino, Giappichelli, 2018.

9 Cfr. da ultimo M. Cammelli, Centro e periferia: l'emergenza fa cadere il velo, in "il Mulino", n. 3, 2020, spec. p. 405, ove ne auspica la "radicale ricostruzione". 
nomici - ed un regionalismo ciclotimico, del quale a fasi alterne si pone in discussione la stessa utilità ${ }^{10}$. Dall'altro, si apprezzano istanze marcatamente centrifughe, nelle quali il centro riesce compresso sia dall'alto, in ragione della capacità di imporsi delle politiche sovranazionali, sia dal basso, a causa di spinte autonomistiche che rivivono nell'aspirazione a gestire ad un livello più prossimo ai consociati funzioni e servizi ricollegati al benessere dei cittadini ${ }^{11}$. Ad una delegittimazione politica ed ordinamentale di alcune istituzioni territoriali si è accompagnata la severa crisi finanziaria, rispetto alla quale sono in corso dinamiche di assestamento ${ }^{12}$, peraltro travolte dagli interrogativi e dall'incertezza che porta con sé l'attuale emergenza pandemica.

È nell'alveo di quanto sommariamente premesso che occorre posizionare le osservazioni che seguono. Esse, nel riguardare principalmente la Regione Lombardia, intendono offrire prospettive di politica del diritto da inquadrarsi, tuttavia, in un più ampio ragionamento sulla natura stessa del ruolo che potrebbe (melius, dovrebbe) assumere il legislatore regionale, anche in altre realtà consimili. Si avrà modo di affrontare i temi (i) dell'esigenza di una normativa regionale di riordino del governo locale, (ii) delle possibilità (o potenzialità) di un riordino nell'attuale fase attraversata dall'impulso verso ulteriori forme e condizioni particolari di autonomia, (iii) del metodo del riordino e del ruolo che dovrebbe assumere il legislatore regionale; non prima, tuttavia, di aver dedicato qualche considerazione alla declinazione del regionalismo vivente, per come finora conosciuto nel nostro Paese e per come, invece, si potrebbe ridefinire in ragione di alcune dinamiche emergenti che valorizzano il principio costituzionale di differenziazione.

${ }^{10}$ In tal senso non stupisce il dibattito sull'istituzione delle macroregioni rivitalizzatosi in Italia negli ultimi anni, che ha conosciuto svolgimenti concreti in altri Paesi nei quali le politiche aggregative approntate hanno condotto alla diminuzione del numero degli enti regionali, come accaduto ad esempio in Francia.

11 È la c.d. "sandwich thesis" cui si riferisce G.F. FERRARI, Federalismo, regionalismo e decentramento del potere in una prospettiva comparata, in "Le Regioni", n. 4, 2006.

${ }^{12} \mathrm{Si}$ veda, diffusamente, S. MANGIAMELI, Le regioni italiane tra crisi globale e neocentralismo, Milano, Giuffrè, 2013. 


\section{Il vecchio e il nuovo: regionalismo dell 'uniformità e regionalismo della differenziazione}

Il regionalismo vive nella vicenda costituzionale italiana ${ }^{13}$ mutuato da una concezione garantista delle autonomie regionali, quale argine a nuove derive autoritarie dello Stato ${ }^{14}$, sotto la copertura del principio autonomistico sancito nell'art. 5 Cost. Declinato nella conformazione del principio di pluralismo istituzionale, esso ha faticato ad affermarsi nonostante la formale previsione delle Regioni in Costituzione: non a caso, le Regioni ordinarie hanno conosciuto una prima attuazione solo dopo vent'anni dall'entrata in vigore della Carta fondamentale e ciclicamente si è dubitato - e ancora si dubita - della bontà o meno dell'istituto regionale ${ }^{15}$. Ciò si deve principalmente alla perdurante tensione, ancora oggi riscontrabile, tra il principio di unità dell'ordinamento - recante esigenze di uniforme sviluppo economico-sociale ed equivalente inveramento dei diritti previsti in Costituzione - e principio autonomistico, cui fa eco il pluralismo istituzionale, ad un tempo forma e formante della società.

Così, tra slanci (incompiuti) verso l'attuazione del regionalismo registratisi negli anni Settanta ${ }^{16}$ e fenomeni, anche marcati, di centralismo di ritorno che hanno attraversato il decennio successivo, è solo negli anni Novanta che la Regione viene definita come "centro propulsore e di coordinamento delle autonomie locali" 17 e si fa luogo alla c.d. "terza regionalizzazione" facente capo alle cc.dd. "Leggi Bassanini"18, nella quale la tensione verso la differenziazione - principio accolto nella legge 59/1997 - non ha visto brillare gli Enti regionali, rimasti in larga parte

13 In verità il dibattito sull'autonomia regionale è molto più risalente rispetto al dato testuale offerto dalla Costituzione repubblicana. A tal proposito, cfr. P.G. GRASSO, Proposte di autonomia regionale agli inizi dell'unità d'Italia, in "Il Politico", n. 2, 1994.

${ }^{14}$ Cfr. V. CRISAFULLI, Vicende della questione regionale, in "Le Regioni”, n. 4, 1982.

${ }^{15}$ Cfr. già M.S. GiANNINI, Del lavare la testa all'asino, in A. BARBERA, F. BASSANINI (a cura), "I nuovi poteri delle regioni e degli enti locali. Commentario al decreto 616 di attuazione della legge 382", Bologna, il Mulino, 1978.

${ }^{16}$ Restano centrali nello studio del fenomeno, soprattutto per quanto attiene al contesto domestico ma anche in chiave comparata, i due volumi ISAP, La regionalizzazione, voll. 1 e 2, Milano, Giuffrè, 1983.

${ }^{17}$ Cfr. la storica sentenza Corte cost. 15 luglio 1991, n. 343

${ }^{18} \mathrm{Su}$ cui si veda, ad es., F.C. RAmpulla, La legge Bassanini e le autonomie locali, in "Il Politico", n. 1, 1997. 
inerti dinanzi alla prospettiva di riallocare in modo differenziato a livello locale le competenze amministrative ${ }^{19}$. A tali dinamiche ha fatto seguito la riforma del Titolo V della parte seconda della Costituzione intervenuta con la legge cost. 3/2001, che ha costituzionalizzato i principi di sussidiarietà, differenziazione ed adeguatezza (art. 118 Cost.).

Anche tale riforma, pur con la necessaria opera di adeguamento del riparto per materie, proprio del regionalismo devolutivo, messa in opera dalla Consulta, non ha condotto alla piena affermazione del disegno regionalista. Da un lato, innanzi alla rigidità del riparto materiale di cui all'art. 117 Cost., la Corte è intervenuta in chiave razionalizzatrice - e, sovente, accentratrice - facendo leva su vari istituti che hanno valorizzato il rilievo delle funzioni e della dimensione dell'interesse sotteso alle materie ${ }^{20}$; dall'altro lato, mentre a livello regionale il raccordo tra Regioni e autonomie locali è stato assicurato dall'istituzione del Consiglio delle autonomie locali $(\mathrm{CAL})^{21}$, a livello centrale il confronto maieutico tra Stato e Regioni è stato affidato alla Commissione parlamentare per le questioni regionali22, in seguito mai attuata. Le istanze di Regioni ed enti locali sono state, dunque, affidate al solo raccordo previsto nel sistema delle Conferenze ${ }^{23}$, il quale tuttavia non sempre ha operato al meglio delle proprie possibilità 24 .

Ancora oggi assistiamo, dunque, ad un sistema nel quale agiscono "regioni senza regionalismo" 25 , cioè senza una vera regionalizzazione

${ }^{19}$ L'opinione è diffusa in dottrina ed ampiamente condivisibile. Cfr., per tutti G. PASTORI, Riordino delle funzioni e redistribuzione a livello locale, in S. GAMBINO, G. FABBRINI (a cura), "Regione e governo locale", Rimini, Maggioli, 1997.

20 Tra i quali, emblematicamente, si possono citare a titolo di esempio la c.d. chiamata in sussidiarietà, la concezione delle cc.dd. materie trasversali o materie-funzione, nonché il c.d. criterio di prevalenza.

${ }^{21}$ Peraltro, con risultati molto differenti, talora deludenti, tra le diverse Regioni.

22 Cfr. l'art. 11 della legge cost. 3/2001.

${ }^{23}$ Nelle sedi proprie del centro "comunitario" cui si riferisce G. SCIULlo, Alla ricerca del centro. Le trasformazioni in atto nell'amministrazione statale italiana, Bologna, il Mulino, 2000.

${ }^{24}$ Così non è accaduto, ad es., durante l'attuale emergenza pandemica. Del resto, come già sottolineato da G. Sciullo, Alla ricerca del centro, cit., p. 148, "[...] le Conferenze rappresentano attualmente succedanei di sedi la cui istituzione rappresenta ormai un'esigenza indilazionabile per un maturo autonomismo. Si intende alludere chiaramente ad organismi del tipo Bundesrat, che il dibattito istituzionale ha variamente denominato".

${ }^{25}$ Nota e plastica locuzione mutuata da G. PASTORI, Le regioni senza regionalismo, in "il Mulino", n. 2, 1980. 
del centro, prima che delle periferie: sicché, solo per esemplificare, permangono Ministeri aventi competenza su materie che sarebbero affidate per Costituzione alla legislazione esclusiva regionale ${ }^{26}$; il Senato della Repubblica risulta nei fatti ancora troppo sganciato dai territori; la riforma del federalismo fiscale ${ }^{27}$, sulla carta ambiziosa ed in linea con il dettato costituzionale, non è mai stata portata a compimento dal punto di vista applicativo ${ }^{28}$; abbiamo assistito, infine, a un sistema delle autonomie locali che ha portato sulle sue spalle l'intero peso della recente crisi economico-finanziaria, in ragione di una normazione statale espressione di un dilagante neo-centralismo ${ }^{29}$.

Questo contesto ordinamentale e culturale ha favorito non già la revisione, bensì il consolidamento di un regionalismo dell'uniformità ${ }^{30}$. Esso, tuttavia, nella traduzione applicativa che ha conosciuto nei fatti, ci ha insegnato che uniformità di statuto giuridico (delle Regioni ordinarie) non equivale ad uniformità nella tutela dei diritti sociali e nell'erogazione delle prestazioni, sicché è accaduto che molte leggi ispirate alla promozione dell'uniformità abbiano prodotto notevoli sperequazioni sociali ${ }^{31}$.

Per questa ragione, è venuta affermandosi una corrente di pensiero incline a sondare le potenzialità insite nella differenziazione ${ }^{32}$, di per

${ }^{26}$ Emblematico il caso dell'agricoltura, che sarebbe competenza residuale delle Regioni ex art. 117, comma 4, Cost.

27 Per un inquadramento teorico v. F. OsCULATI, Il federalismo fiscale nella prospettiva della scienza delle finanze, in G.F. FERRARI (a cura), "Federalismo, sistema fiscale, autonomie. Modelli giuridici comparati”, Roma, Donzelli, 2010.

${ }^{28} \mathrm{Ci}$ si riferisce alla mancata - o solo parziale - attuazione dell'art. 119 Cost. e della legge 42/2009. A tal proposito si veda almeno L. ANTONINI, Un requiem per il federalismo fiscale, in "Federalismi.it", n. 16, 2016; L. Antonini, Federalismo all'italiana. Dietro le quinte della grande incompiuta, Venezia, Marsilio, 2013.

${ }^{29}$ G.C. De MARTIN, Il disegno autonomistico disatteso, tra contraddizioni e nuovi scenari problematici, in "Istituzioni del Federalismo", n. 1, 2014; S. MANGIAMELI, Le regioni italiane tra crisi globale e neocentralismo, cit., passim.

30 Peraltro occorre rilevare che il medesimo problema si poneva anche nei rapporti tra Regioni ed Enti locali, influenzati dalla forza attrattiva della Regione. Sul punto, si veda F.C. RAMPULLA, La politica locale nel sistema istituzionale italiano, in "Il Politico", n. 1, 1984.

31 Approfondisce questo aspetto C. PINELl, Mercati, amministrazioni e autonomie territoriali, Torino, Giappichelli, 1999, passim, spec. p. 275.

${ }^{32} \mathrm{Si}$ veda, emblematicamente, per tutti, L. ANTONINI, Il regionalismo differenziato, Milano, Giuffrè, 2000. 
sé coessenziale al regionalismo ed al concetto stesso di autonomia ${ }^{33}$. Già negli anni Novanta - come si è detto - si sono fatte largo simili istanze nel sistema delle autonomie, poi confermate nel dettato costituzionale del 2001, ove il principio di differenziazione è stato posto accanto a quello di sussidiarietà e di adeguatezza, quale declinazione specializzata del principio di eguaglianza sostanziale ${ }^{34}$. Tali istanze riecheggiano attualmente nelle recenti richieste di ulteriori forme e condizioni particolari di autonomia ex art. 116, comma 3, Cost. di Lombardia, Veneto ed Emilia-Romagna. Si tratta di un istituto ricompreso nella novella del Titolo $\mathrm{V}$ al fine di mitigare la rigidità del riparto materiale ivi accolto e, laddove attivato, correggerne le conseguenze ${ }^{35}$, secondo una prospettiva tale da riconfermare, ancora una volta, che regionalismo significa, anzitutto, differenziazione.

Evidentemente il percorso che oggi si affaccia all'orizzonte, imperniato sulla possibile attivazione della menzionata norma costituzionale ${ }^{36}$, riesce di complicata attuazione ma rivela tutta l'inquietudine di Regioni in costante ricerca di autonomia. Un'autonomia, cionondimeno, che bisogna dimostrare di saper meritare sul campo, alla prova dei fatti, esplicitata nella vocazione differenziante intrinsecamente connaturata all'azione regionale, anzitutto sul fronte dell'esercizio della potestà legislativa.

Quest'ultimo rappresenta il fronte avanzato di una diffusa critica alle Regioni ${ }^{37}$, che per molto tempo alcune di esse - tra le quali, ad

${ }^{33}$ Già un secolo fa si metteva in discussione il paradigma dell'uniformità con riferimento ai Comuni: cfr. a riguardo U. BORSI, Regime uniforme e regime differenziale nell'autarchia locale, in "Rivista di diritto pubblico", n. 1, 1927.

${ }^{34}$ In ragione del quale situazioni diverse devono essere trattate e disciplinate in modo differenziato. Così R. BIN, Chi ha paura delle autonomie?, in AA.VV., "Scritti in ricordo di Paolo Cavaleri”, Napoli, ESI, 2016, p. 52.

${ }^{35}$ Cfr., ex multis, G. Falcon, Il nuovo Titolo V della Parte seconda della Costituzione, in "Le Regioni", n. 1, 2001.

${ }^{36}$ Le implicazioni relative all'applicazione della norma costituzionale sono state oggetto di una letteratura imponente sulle principali riviste scientifiche del settore. Per una prima ricognizione di tali profili, anche in relazione alle materie oggetto di negoziazione, cfr. ad es. L. Violini, L'autonomia delle Regioni italiane dopo i referendum e le richieste di maggiori poteri ex art. 116, comma 3, Cost., in "Rivista AIC", n. 4, 2018.

${ }^{37} \mathrm{Cfr}$. il notevole contributo di M. CAMMELLI, Regioni e regionalismo. 1948-2013, Napoli, Editoriale Scientifica, 2014, già pubblicato in versione ridotta con il titolo Regioni e regionalismo: la doppia impasse, in "Le Regioni", n. 4, 2012, numero monografico 
esempio, l'Emilia-Romagna, la Toscana, il Veneto e la Lombardia hanno saputo mitigare e a tratti respingere ${ }^{38}$ congegnando leggi regionali all'avanguardia nei più svariati settori. È su queste basi, quindi, che occorre muoversi per intravedere e riconsiderare alcune esigenze di riforma che proprio in Lombardia meritano attenzione a tal punto da risultare decisive per il futuro dell'intero sistema regionale delle autonomie ma la cui trattazione, almeno sinora, è stata inspiegabilmente rinviata da parte dei decisori politici di legislatura in legislatura, tradendo un atteggiamento timido ed attendista, comunque non in linea con la tradizione di una Regione che ha fatto dell'innovazione normativa il proprio vessillo per decenni ${ }^{39}$. Le considerazioni che seguono, peraltro, prendendo in esame il caso studio lombardo, in verità possono essere estese anche a molte altre Regioni italiane, dal momento che alcune delle criticità osservate presentano carattere generalizzato e sono direttamente ricollegabili alla mancata riforma costituzionale del 2016.

\section{Esigenze di riordino del governo locale e ruolo della Regione. Il caso della Lombardia}

Cerchiamo, dunque, di inquadrare come si atteggia la realtà territoriale lombarda rispetto alle dinamiche nazionali. Il contesto nel quale ci si muove - come anticipato supra, par. 1 - rivela un panorama nazionale delle autonomie frammentato e perplesso. La riforma territoriale inaugurata con la c.d. "Legge Delrio" è stata, dapprima, "interrotta" dalla mancata riforma costituzionale che ne avrebbe dovuto costituire la logica premessa concettuale, non l'epilogo formale 40 ; in seguito, essa è stata ul-

recante gli interventi di G. Falcon, S. Bartole, R. Bin, P. Caretti, U. De Siervo, F. MerLONI, V. Onida, D. Sorace, G. TARLi Barbieri e L. Violini.

${ }^{38} \mathrm{La}$ critica concernente gli spazi di legislazione regionale rimasti inutilizzati emerge chiaramente anche in L. VANDELLI, Introduzione. Il federalismo alla prova: le regioni tra uniformità e differenziazione, in L. VANDELLI, F. BASSANINI (a cura), "Il federalismo alla prova: regole, politiche, diritti nelle regioni”, Bologna, il Mulino, 2012.

${ }^{39} \mathrm{Si}$ pensi alla legge reg. 31/1997, poi seguita dalle leggi reg. 33/2009 e 23/2015 in materia di servizio sanitario regionale, oppure alla recente legge reg. 11/2014 in materia di attività e libertà d'impresa.

$40 \mathrm{Il} \mathrm{metodo}$ adottato risulta assai criticabile, benché richiami quanto già era accaduto con le c.d. "Leggi Bassanini", approvate a costituzione invariata e seguite dalla riforma costituzionale approntata con la legge cost. 3/2001. 
teriormente disattesa dagli interventi legislativi successivi, principalmente informati al taglio lineare delle risorse poste a presidio dell'esercizio delle funzioni provinciali e metropolitane, peraltro in un quadro di sempre maggiori sollecitazioni gravanti sui Comuni ${ }^{41}$.

Assistiamo all'esigenza di ricondurre ad unità un quadro delle autonomie che, in caso contrario, rischia di degenerare nella costante perdita di legittimazione politico-istituzionale e di capacità amministrativa e gestionale nell'erogazione di servizi alla cittadinanza secondo criteri di efficienza, efficacia ed ottimalità quantitativo-qualitativa ${ }^{42}$.

Questa deriva coinvolge sia gli Enti aventi vocazione di area vasta (Province e Città metropolitane) che i Comuni di tutte le Regioni italiane. Tuttavia, è appena il caso di sottolineare il fatto che in un territorio complesso ed eterogeneo come la Lombardia, sul quale insistono una moltitudine di soggetti istituzionali e funzionali, la deriva anche di un solo comparto rischia di trascinare con sé l'intera Regione: del resto, come è stato detto in passato, in tutte le Regioni d'Italia è presente "un Mezzogiorno"43 e a tale realtà non sfugge nemmeno la Lombardia, nella quale vi sono dinamiche socio-economiche preoccupanti che interessano intere Province.

Da qui la necessità che il legislatore assuma un ruolo attivo ed incisivo. L'auspicio di un rinnovato protagonismo regionale muove non solo dal contesto delineato in precedenza, ma anche dal fatto che la Lombardia condivide con altre Regioni settentrionali 44 uno spiccato policentrismo amministrativo, frangente che concorre a rendere maggiormente complesso - ma, allo stesso tempo, ambizioso - il possibile riordino territoriale delle autonomie infraregionali. Per esemplificare,

\footnotetext{
${ }^{41}$ Sul punto, si veda F. Osculati, La dotazione organica e finanziaria degli Enti di area vasta. Un assetto ancora provvisorio ovvero una vicenda dirigistica, cit..

${ }^{42} \mathrm{Cfr}$. le acute osservazioni presenti in F. AnNovazzI, F. OsculaTI, La trasformazione delle Province. Poca politica, tanto asfalto, in "Astrid Rassegna”, n. 6, 2015.

${ }^{43}$ Segnatamente Passerin d'Entrèves ebbe a dire che: "C'è in ogni Regione settentrionale un 'Mezzogiono'” (E. PASSERIN D'EnTRÈVES, Gli aspetti storici degli squilibri regionali, in AA.VV., "Gli squilibri regionali e l'articolazione dell'intervento pubblico", Atti del Convegno di studio svoltosi a Torino e a Saint Vincent dal 3 al 7 settembre 1961, Milano, Lerici Editori, 1962, p. 265, evento di cui è presente un resoconto anche in "Il Politico", n. 3, 1961).

${ }^{44} \mathrm{Cfr}$., ad es., la Regione Piemonte, nella quale vi sono 1.181 Comuni, alcuni dei quali tali da rappresentare polarità urbane di sicuro rilievo per dimensione e vocazione istituzionale.
} 
oggi insistono in Lombardia ben 1.506 Comuni, 11 Province e 1 Città metropolitana, 23 Comunità montane, quasi 70 Unioni di Comuni ${ }^{45}$ ma operano, altresì, 8 Agenzie di Tutela della Salute (ATS), nonché svariate Aziende Socio Sanitarie Territoriali (ASST), Camere di Commercio (CCIAA) ${ }^{46}$, Consorzi di Bonifica, Consorzi di Bacino imbrifero montano, Parchi regionali; e sarebbe possibile continuare a lungo nell'elencazione di Enti ed Agenzie che si trovano a presidiare territori tra loro molto diversi e che rischiano sempre più di evolvere secondo geometrie e velocità variabili, incrementando in modo preoccupante i divari territoriali. A fronte di una moltitudine di soggetti istituzionali e funzionali, l'esistente giuridico propone una corrispondente moltitudine di azzonamenti ed ambiti di esercizio delle funzioni amministrative che pertengono a tali soggetti, sicché le funzioni da esercitare assurgono ad elemento centrale nel ragionamento sul possibile riordino del governo locale.

Preso atto della presenza di un reticolo territoriale difficilmente districabile, il legislatore regionale potrebbe forse spingersi fino a puntare ad una difficile e complicata semplificazione, la quale tuttavia sarebbe difficilmente conseguibile nel breve o medio periodo; oppure potrebbe seguirsi, semmai, il metodo di un più forte coordinamento tra i vari livelli di governo e amministrazione, a loro volta presidiati da soggetti istituzionali, organizzativi e funzionali ${ }^{4}$.

Il rischio di incorrere in un "effetto trascinamento" risulta amplificato in una Regione autorevole quale è la Lombardia, la quale si è distinta nei cinquant'anni di attività per il fatto di aver saputo ergersi a laboratorio di sperimentazione di buone prassi amministrative e, prima

${ }^{45}$ Ci si riferisce sia alle Unioni istituite ai sensi della Legge reg. 19/2008 e censite nell'apposito registro di cui all'art. 20 bis della medesima legge regionale (le cosiddette Unioni di Comuni Lombarde-UCL), sia alle Unioni di Comuni istituite ai sensi dell'art. 32 del TUEL.

${ }^{46}$ Queste ultime, peraltro, sono state oggetto di una riorganizzazione volta alla ridefinizione delle circoscrizioni territoriali ed al successivo accorpamento iniziata con il D.lgs. 219/2016. Per alcune realtà specifiche, tra cui quella che coinvolge anche la costituenda Camera di Cremona, Mantova e Pavia, la transizione istituzionale non è ancora giunta a conclusione, anche a causa dei ricorsi promossi per osteggiare il richiamato accorpamento.

${ }^{47} \mathrm{Si}$ veda amplius, se si vuole, le proposte descritte in G.C. RICCIARDI, Un paradigma sperimentale di integrazione nell'Area vasta: i raccordi tecnici con i soggetti funzionali et similia, in G.C. RICCIARDI, A. VENTURI (a cura), "La riorganizzazione territoriale e funzionale dell'Area vasta", Torino, Giappichelli, 2018. 
ancora, di buone leggi regionali, recanti soluzioni in seguito replicate sul territorio nazionale. Non deve stupire, quindi, che in una delle Regioni costituenti i 4 Motori dell'Europa vi sia una provincia, come Pavia $^{48}$, che si classifica oltre $1^{\prime} 800$ esimo posto 49 tra le aree vaste europee più sviluppate dal punto di vista dei principali indicatori economico-sociali ${ }^{50}$. Nella sua crudezza, questo dato rappresenta un divario inter-territoriale significativo, che necessita di un presidio forte da parte della Regione e, in particolare, di politiche perequative e di raccordo tecnico tra Enti finalizzate all'inclusione delle aree più in sofferenza ${ }^{51}$, entro un quadro cooperativo di più ampio respiro. Questo disegno può tuttavia concretizzarsi solo attraverso la concezione di una normativa all'avanguardia, recante un'idea chiara di riorganizzazione del governo locale avente ad oggetto sia l'esito di una possibile riforma sia, ancor prima, il metodo.

Per vero - come si è detto supra, par. 2 - quanto alle condizioni propedeutiche all'attuazione di tale prospettiva, l'atteggiamento del legislatore regionale è stato finora sin troppo attendista.

Sorprende, infatti, che la Lombardia mantenga, ancora oggi, una normativa come la legge reg. 19/2008 in tema di "Riordino delle Comunità montane della Lombardia, disciplina delle unioni di comuni lombarde e sostegno all'esercizio associato di funzioni e servizi comunali", più volte interpolata pur senza modificare l'impostazione, a tratti vetusta, del dettato

${ }^{48}$ Cfr. UnIVERSità Degli StUdi di PAVIA, Sintesi del Documento d'Indagine per lo sviluppo di un sistema Milano-Pavia, dossier recante la sintesi del documento presentato al Convegno "Per lo sviluppo del sistema Milano-Pavia", 26 Settembre 2018, in https://www.assolombarda.it/governance/sede-di-pavia/progetti/per-lo-sviluppo-di-un-sistema-pavia-milano, p. 12, ove la provincia di Pavia appare come l'ultima della Lombardia per PIL pro-capite, recando valori più che dimezzati rispetto alla città metropolitana di Milano.

${ }^{49} \mathrm{Ci}$ si riferisce al dato di fonte EUROSTAT sulla generazione di valore aggiunto nelle articolazioni NUTS-3. Rispetto a queste ultime, nel 2016 il dato pro-capite della provincia di Pavia si assesta alla posizione 831 su un totale di 1.346 circoscrizioni comparabili dell'Unione Europea, manifestando un trend in declino, dovuto alla perdita di 384 posizioni negli ultimi 13 anni, come rilevato nella ricerca CONFINDUSTRIA PAVIA, UNIVERSITÀ DEGLI STUDI DI PAVIA, La competitività del territorio pavese nel sistema metropolitano regionale, in https://www.assolombarda.it/governance/sede-di-pavia/progetti/ricerca-completa-lacompetitivita-del-territorio-pavese-nel-sistema-metropolitano-regionale, p. 5.

${ }^{50}$ Il dato è ampiamente rappresentativo dell'evidente vulnerabilità dell'area.

${ }^{51}$ La difficoltà di alcune zone svantaggiate della Regione nel far fronte ai bisogni palesati dalle rispettive comunità appare particolarmente significativa per i piccoli Comuni, come suggerisce A. Ceriani, Piccoli Comuni in Lombardia, in “Amministrare”, n. 1, 2009. 
normativo. Si tratta di un testo concepito ben prima delle molte riforme che, a partire dal d.1. 78/201052 (cd. "decreto Monti"), si sono susseguite nell'ultimo decennio interessando gli Enti di prossimità e non solo.

Basti pensare, inoltre, alla "resilienza" delle Comunità montane, le quali conservano funzioni, ruolo e status - per ragioni sia storiche, sia geo-morfologiche - tali da giustificarne il mantenimento se non addirittura il rilancio ma che esigerebbero misure e strumenti più stringenti di controllo della spesa pubblica e di coordinamento istituzionale e funzionale con la Regione ${ }^{53}$.

Soprattutto, si pensi alla logica generale che presiede alla normativa in materia di forme associative intercomunali la quale, a fronte di una struttura del governo locale di derivazione napoleonica, dovrebbe probabilmente accogliere alcune soluzioni mutuate dall'esperienza transalpina: in Francia, infatti, pur in presenza di circa 35.000 Comuni, la quasi totalità di essi agisce entro logiche di cooperazione interlocale, a differenza di quanto accade nel nostro contesto domestico; frangente, questo, tale da sottolineare ancora una volta che il problema non attiene tanto al numero dei Comuni - e non riguarda solo la loro dimensione - quanto alle modalità di esercizio delle funzioni loro intestate ed ai paradigmi organizzativi in concreto adottabili per farvi fronte.

In una logica di razionalizzazione della spesa pubblica, permane il problema della multipla incentivazione, a vario titolo giuridico, dei medesimi territori ${ }^{54}$. Ciò appare evidente in certe zone montane, ove i Comuni svantaggiati percepiscono risorse a titolo dello svantaggio ma aderiscono contemporaneamente a Unioni di Comuni candidabili alla contribuzione regionale prevista in caso di iscrizione in apposito registro $^{55}$, a loro volta operanti all'interno di Comunità montane, le quali,

52 Decreto successivamente convertito con modificazioni in legge 122/2010.

53 V. amplius, A. CERIANI, G.C. RiCCIARDI, Le Comunità montane come forme associative, Pavia-Milano, Pavia University Press, 2020. Per una rassegna su alcune normative regionali vigenti cfr. G. MARCHETTI, A favore delle zone montane: dalla legislazione organica statale a legislazioni organiche regionali (e oltre), in "Le Regioni", n. 5, 2020.

${ }^{54}$ Tema sul quale si veda la pregevole ricostruzione svolta da A. ZATTI, Costi e benefici dei processi aggregativi e il ruolo delle politiche incentivanti, in G.C. RICCIARDI, A. VENTURI (a cura), "Unioni e fusioni di Comuni. Elementi teorici e prassi operative. Volume I. L'Unione di Comuni come progetto multidimensionale", Rimini, Maggioli, 2020.

${ }^{55} \mathrm{Si}$ veda. supra, nota 45 . L'iscrizione nel registro è subordinata all'esercizio in forma associata di almeno cinque funzioni fondamentali comunali. 
oltre al "contributo di funzionamento" di matrice regionale, percepiscono le risorse mutuate dal fondo statale regionalizzato previsto a favore delle forme associative ${ }^{56}$. Al di là della dispersione di risorse, per vero marginali rispetto ai volumi dei bilanci degli enti locali interessati ${ }^{57}$, il problema attiene al fatto che alcune misure si pongono su un piano teleologico antitetico le une rispetto alle altre, con la conseguenza di elidersi vicendevolmente quanto agli esiti attesi ${ }^{58}$.

Sul fronte degli azzonamenti ${ }^{59}$ sorprende, inoltre, che - nonostante molti tentativi in tal senso ed il laconico richiamo alla zona omogenea per i Comuni della Comunità montana nonché al territorio dell'insieme dei Comuni afferenti a ciascuna azienda sociosanitaria territoriale ${ }^{60}-$ in Lombardia non sia finora mai stato attuato l'art. 33 del TUEL. La norma imporrebbe alla Regione di individuare i livelli ottimali di esercizio associato delle funzioni amministrative dei Comuni di minore dimensione ed esigerebbe che la Regione predisponesse un programma di individuazione degli ambiti per la gestione associata di funzioni e servizi, realizzato anche - ma non necessariamente - attraverso Unioni di Comuni. Infatti, non ci si riferisce a un'organizzazione fondata sull'esercizio delle funzioni mediante Unioni di Comuni ma, al contrario,

${ }^{56}$ Sul punto, sia consentito il richiamo a G.C. RicCIARd, A. ZatTI, Politiche incentivanti e riordino territoriale, in G.C. RicCIARDI, A. VENTURI (a cura), "Unioni e fusioni di Comuni. Elementi teorici e prassi operative. Volume I. L'Unione di Comuni come progetto multidimensionale", Rimini, Maggioli, 2020, nonché gli altri approfondimenti raccolti nella Parte II, Sezione II, del medesimo volume.

57 Per una dettagliata indicazione dei volumi finanziari e del contesto normativo, cfr. GiUnTA Regionale Della lombardia, Deliberazione XI/3009 del 30 marzo 2020, ed in particolare l'Allegato recante la Relazione triennale al Consiglio regionale dal titolo "Stato di attuazione e risultati ottenuti nella diffusione dell'esercizio di funzioni e servizi comunali attraverso forme stabili di Gestione Associata. Annualità 2017-2019", elaborata da Regione Lombardia con il supporto tecnico-scientifico del Dipartimento di Scienze Politiche e Sociali dell'Università di Pavia.

${ }^{58} \mathrm{Cfr}$., ancora A. ZATTI, Costi e benefici dei processi aggregativi e il ruolo delle politiche incentivanti, cit..

${ }^{59} \mathrm{Il}$ tema era già stato autorevolmente affrontato in passato, con riferimento alle associazioni di Comuni, da F.C. RAmpulla, Le associazioni di comuni tra autonomia locale e autarchia regionale, Padova, Cedam, 1984.

${ }^{60} \mathrm{Cfr}$. artt. 1, comma 4 e 17, comma 2, della Legge reg. 19/2008. Accreditando una lettura riduttiva dell'art. 33 del TUEL, potrebbe ritenersi che la norma sia stata attuata mediante il richiamo ai due ambiti menzionati nel testo; si tratterebbe, tuttavia, di una concezione meramente formale del processo attuativo, del tutto insufficiente rispetto alla ratio sottesa alla norma stessa ed al ruolo regionale che la sua piena attuazione richiederebbe. 
alla costituzione di Zone omogenee anche nelle zone di pianura, ove l'assenza di un alter ego delle Comunità montane evidenzia una richiesta di governance delle amministrazioni di prossimità ad oggi rimasta insoddisfatta, da svolgersi mediante un apposito livello organizzativo ma non obbligatoriamente attraverso la costituzione di un Ente locale ${ }^{61}$.

In Lombardia, come in molte Regioni italiane, sono state successivamente adottate normative di attuazione della c.d. "Legge Delrio" quali la Legge reg. 19/2015 e la Legge reg. 32/2015 - che contengono anche soluzioni interessanti, ma non hanno affrontato - e, quindi, tantomeno risolto - il problema del raccordo tra la congerie di soggetti pubblici e funzionali cui abbiamo fatto cenno in precedenza. Naturalmente anche su quest'ultimo profilo occorrerebbe che una legge della Regione disciplinasse l'insieme delle questioni esposte in una logica di connessione tra contenuti interdipendenti: in una recente ricerca promossa dal Dipartimento di Scienze Politiche e Sociali dell'Università di Pavia (DSPS) ${ }^{62}$ - che, storicamente, si situa nel solco di una tradizione inaugurata con un'altra importante ricerca commissionata proprio dalla Regione Lombardia ${ }^{63}$ - si discorre diffusamente di questi aspetti e di come le richiamate istanze di coordinamento possano contemperarsi tra loro, con forme snelle e leggere, a patto che vi sia una regia regionale ${ }^{64}$.

Dal quadro laconicamente delimitato emerge lampante la necessità di porre mano ad una normativa che necessita adattamenti e ripensamenti; essa, tuttavia, non può prescindere da un accompagnamento autorevole della Regione, la quale dovrebbe tornare a guidare il processo endogeno di riordino abbandonando l'attesa di un legislatore

${ }^{61}$ Anzi, sul punto, chi scrive opterebbe per un modello leggero a carattere meramente organizzativo, peraltro già autorevolmente sostenuto in passato. Sul punto v. infra, nt. 78 .

$62 \mathrm{Si}$ veda G.C. RICCIARDI, A. VENTURI (a cura), La riorganizzazione territoriale e funzionale dell'Area vasta, Torino, Giappichelli, 2018.

${ }^{63}$ Il pensiero corre alla pionieristica ricerca coordinata da U. РотоTSCHNIG e promossa dall'Università di Pavia, dal titolo AA.VV., Legge generale sull'amministrazione locale, $\mathrm{Pa}$ dova, Cedam, 1977, commentata da P.G. GRASSO, Profili costituzionali della riforma della legge comunale e provinciale. Note ad una proposta di legge, in "Il Politico", n. 3, 1978.

${ }^{64} \mathrm{Ci}$ si riferisce alla possibilità di istituire tavoli di coordinamento orizzontale e verticale, a carattere infraregionale e infraprovinciale, declinata in F.C. RAMPULLA, G.C. RICCIARDI, Schema normativo, nonché in G.C. RICCIARDI, Un paradigma sperimentale di integrazione nell'Area vasta: i raccordi tecnici con i soggetti funzionali et similia, cit., entrambi in G.C. RicCIARDI, A. VENTURI (a cura), "La riorganizzazione territoriale e funzionale dell'Area vasta", Torino, Giappichelli, 2018. 
statale che difficilmente provvederà, in tempi rapidi, a sciogliere i nodi della normativa nazionale. Si tratta di un approdo che riguarda, naturalmente, anche le altre Regioni nelle quali la richiesta di governance dal basso non può essere affrontata solamente mediante politiche settoriali ma richiede un atteggiamento propositivo e coraggioso del legislatore regionale.

\section{Alcune inferenze tra (possibile) attuazione del regionalismo differenziato e riorganizzazione delle autonomie infraregionali}

Preso atto dell'esistenza di un'esigenza diffusa di riordino del governo locale - riguardante, in primo luogo, la Lombardia ma tale da coinvolgere anche altri Enti omologhi - giova chiedersi quale possa essere l'impatto del regionalismo differenziato nel sistema regionale e come possa atteggiarsi il legame con gli Enti locali infraregionali nonché, da ultimo, quale metodo occorra seguire nella conseguente riorganizzazione del governo locale ${ }^{65}$. Infatti la sfida rappresentata dal regionalismo differenziato rivela profili ricostruttivi particolarmente ampi e profondi.

Giova anticipare sin d'ora che le esigenze di una nuova disciplina giuridica del governo locale lombardo persistono sia che vada in porto il percorso relativo all'attuazione dell'art. 116, comma 3, Cost., sia che esso subisca una battuta d'arresto. Infatti, al di là delle richieste competenziali che afferiscono all'asimmetria, viene in gioco il ruolo - o, forse, sarebbe meglio dire la resilienza ordinamentale - delle Regioni e del regionalismo stesso, che in questa stagione possono tentare un deciso rilancio verso nuove forme di governo territoriale, sia nell'ambito delle Regioni richiedenti l'asimmetria, sia all'interno di una dimensione più ampia, di carattere interregionale ${ }^{66}$.

La strada che si propone in questa sede, valevole per tutte le Regioni,

${ }^{65}$ I profili brevemente rappresentati in questo paragrafo vengono diffusamente analizzati, se si vuole, in G.C. RICCIARDI, La dimensione amministrativa del regionalismo asimmetrico. Considerazioni di teoria generale, in "Federalismi.it", n. 25, 2020, al quale si rimanda per gli approfondimenti logico-giuridici e per tutti i riferimenti bibliografici.

${ }^{66} \mathrm{Cfr}$. A. Venturi, La proiezione interregionale dell'autonomia, in L. Violini, Q. CAMERLENGo (a cura), "Lineamenti di diritto costituzionale della Regione Lombardia", Torino, Giappichelli, 2014. 
è quella di incentrare il ragionamento sulle funzioni amministrative. Una riforma territoriale fondata sul rilievo delle funzioni - e del loro esercizio ridistribuito tra i livelli di governo in ragione dei principi di sussidiarietà, differenziazione ed adeguatezza - costituisce una delle poche soluzioni di ingegneria istituzionale in grado di liberare risorse e sfruttare al meglio le potenzialità intrinseche all'effettiva attuazione dell'art. 118 Cost., anche rispetto all'occasione rappresentata dal regionalismo asimmetrico.

$\mathrm{Ci}$ si potrebbe chiedere per quale motivo, nell'accennare al regionalismo differenziato, si stia facendo riferimento all'art. 118 Cost. La prima risposta al quesito deriva dal fatto che tale norma - concernente l'allocazione delle funzioni amministrative tra i livelli di governo territoriale - pur assumendo un ruolo centrale in qualsiasi ragionamento incentrato sulla ridistribuzione delle funzioni amministrative, è rimasta in larga parte inattuata nei primi vent'anni di applicazione del nuovo Titolo V della Costituzione ${ }^{67}$. Se da un lato, dunque, la riorganizzazione delle autonomie infraregionali incentrata sulla tassonomia delle funzioni non può prescindere dall'attuazione dell'art. 118 Cost., dall'altro lato il richiamo ai principi di sussidiarietà, differenziazione e adeguatezza ivi menzionati risulta conferente anche per un'altra ragione. Per vero, quando si discorre dell'attuazione dell'art. 116, comma 3, Cost. ci si riferisce anche all'esercizio delle funzioni correlate alla distribuzione competenziale di cui all'art. 118 Cost. Infatti, non essendo stato esplicitato nell'art. 116, comma 3, Cost. un univoco riferimento letterale alle competenze legislative, nulla vieta che mediante l'intestazione delle materie di cui agli artt. 117, comma 3, Cost. e 117, comma 2, lett. l), n) e $s$ ), la Regione destinataria divenga titolare sia di competenze legislative sia amministrative ${ }^{68}$, come è stato correttamente inteso anche da Regione Lombardia nella conduzione dei negoziati con il Governo. La scelta regionale di declinare in 131 funzioni le 20 materie sulle quali è stata richiesta l'asimmetria ${ }^{69}$ conferma che occorre spostare l'atten-

${ }^{67}$ Parla addirittura di "oblio" dell'art. 118, comma 1, Cost., ad es., M. CAMmELLI, Flessibilità, autonomia, decentramento amministrativo: il regionalismo oltre l'art.116.3 Cost., in "Astrid Rassegna", n. 10, 2019.

${ }^{68}$ Ciò avverrebbe, in prima battuta, secondo il modello del c.d. parallelismo delle funzioni, che in tal caso troverebbe applicazione esclusivamente rispetto agli ambiti materiali costituenti il titolo della "specialità ponderata" delle Regioni ordinarie richiedenti le ulteriori forme e condizioni particolari di autonomia.

${ }^{69} \mathrm{Ci}$ si riferisce alle prime bozze di intesa redatte per individuare e regolare il catalogo 
zione dal catalogo delle materie all'esercizio delle funzioni amministrative, anche perché la vera differenziazione regionale - attese le strette maglie entro cui riesce confinata la potestà legislativa regionale - sinora è stata quella mutuata dalle amministrazioni regionali a livello amministrativo.

Se il regionalismo amministrativo costituisce la via italiana alla differenziazione, ne deriva che il vero cuore del regionalismo differenziato - a prescindere dal dato letterale inferibile dalla Costituzione, avente ad oggetto le materie e dall'opinione dominante, che si richiama alla potestà legislativa ritenendo marginale quella amministrativa - potrebbe ricercarsi soprattutto nell'esercizio di "ulteriori” funzioni amministrative rivendicabili, piuttosto che nella possibilità della Regione di legiferare nelle materie oggetto delle ulteriori forme e condizioni particolari di autonomia. Queste ultime, paradossalmente, potrebbero risolversi eventualmente anche solo in innovativi paradigmi di esercizio delle funzioni correlate alle materie di competenza concorrente, oppure, potrebbero sostanziarsi nell'esercizio tout court di nuove funzioni: in ogni caso, saremmo di fronte ad un'innovazione sostanziale di rilievo rispetto all'attuale tassonomia delle prerogative regionali.

Se si muove da questi presupposti, non è difficile immaginare che il momento più gravoso, all'indomani del conferimento regolato dall'intesa, possa essere quello della ridistribuzione funzionale. Da questo punto di vista, la prospettiva dell'asimmetria prelude necessariamente ad una valorizzazione del tessuto regionale del governo locale, dal momento che alcune funzioni oggetto di conferimento ai sensi dell'intesa potrebbero essere ridistribuite sul sistema degli Enti locali, a maggior ragione in una Regione come la Lombardia conno-

di materie sulle quali la Regione Lombardia richiedeva al Governo ulteriori forme e condizioni particolari di autonomia, nelle quali alle 20 materie oggetto della richiesta si ricollegavano ben 131 funzioni amministrative con la finalità di stabilire il contenuto concreto delle "condizioni particolari" di autonomia. La scelta si fondava sul presupposto che la richiesta sulla singola materia non comportasse la devoluzione della materia nel suo complesso ma solo quelle funzioni ad essa ricollegabili utilmente allocabili al livello regionale. Questa interpretazione "amministrativa" del regionalismo differenziato era stata in un certo senso anticipata, pur escludendo il passaggio della potestà legislativa, già da V. CERULLI IRELLI, Gestioni comuni tra Regioni e modelli di regionalismo differenziato, in A. MAStromarino-J.M. CASTELlà ANDREU (a cura), "Esperienze di regionalismo differenziato. Il caso italiano e quello spagnolo a confronto", Milano, Giuffrè, 2008. 
tata da inespresse potenzialità di raccordo interno del sistema multilivello delle autonomie locali. Occorre quindi riflettere sulle modalità di plausibile applicazione amministrativa ed organizzativa connessa alle maggiori competenze richieste e muovere da un'approfondita istruttoria relativa alla sussistenza della capacità amministrativa del sistema regionale e delle autonomie infraregionali di far fronte ai nuovi compiti aggiunti.

Infatti, già negli anni Cinquanta Massimo Severo Giannini, ammoniva che "in principio sono le funzioni" "70. Con questa espressione il Maestro riteneva di fornire anzitutto un orizzonte metodologico con riferimento a qualsiasi riforma o riorganizzazione della pubblica amministrazione, esprimendo il principio per cui le scelte organizzative dovessero seguire l'assetto delle funzioni ${ }^{71}$.

La questione interessa anche il regionalismo differenziato, dal momento che le ulteriori competenze sono necessariamente sempre seguite da ulteriori funzioni e, con esse, da rinnovate esigenze di strutturazione degli uffici e delle risorse umane ${ }^{72}$, finanziarie e strumentali necessarie ai fini di far fronte ai nuovi compiti assunti volontaristicamente dalla Regione richiedente.

Qualora il percorso di differenziazione dovesse andare a buon fine, sarebbe allora necessario valorizzare gli altri livelli di governo giungendo a considerare anche il ruolo epicentrico delle cc.dd. "Città

70 M.S. GianNINI, In principio sono le funzioni, in Amm. civ., n. 1, 1957.

${ }^{71} \mathrm{Il}$ medesimo principio venne in seguito ripreso e sviluppato autorevolmente anche da altri commentatori, tra i quali U. PотоTSCHNIG, La delega di funzioni amministrative regionali agli enti locali, in "Foro amministrativo", n. 3, 1971, ora in ID., Scritti scelti, Padova, Cedam, 1999, spec. pp. 502-504, ove nell'analizzare l'art. 118 Cost. nella formulazione allora vigente, ravvisava la possibilità di " [...] dar vita ad una organizzazione amministrativa di tipo nuovo e moderno [...] in cui la scelta delle strutture organizzative da impiegare segue, e non precede, la disciplina dell'azione da svolgere". Peraltro resta insuperata, ai fini della comprensione dei caratteri della delega di funzioni e del rapporto tra queste ultime e l'organizzazione ovvero l'utilizzazione degli uffici, l'opera di F. RoVERSI MONACO, La delegazione amministrativa nel quadro dell'ordinamento regionale, Milano, Giuffrè, 1970. Sulla relazione intercorrente tra organizzazione e funzioni si veda anche, più di recente, G. SciUllo, L'organizzazione amministrativa. Principi, Torino, Giappichelli, 2013.

${ }^{72}$ Cfr. S. CASSESE, Il sofisma della privatizzazione del pubblico impiego, in "Rivista italiana di diritto del lavoro", n. 3, 1993, p. 292, ove l'autore rammenta che "[...] quelli del personale sono problemi seguaci, nel senso che debbono adattarsi al tipo di funzioni ed organizzazioni prescelti”. 
medie". Tale soluzione rappresenterebbe un'applicazione concreta del principio di differenziazione ed avrebbe il pregio di disinnescare i timori di eccessiva rigidità del percorso devolutivo-funzionale, ascrivendo alla dimensione sovracomunale (Città metropolitane e Province) ed intercomunale (forme associative intercomunali ${ }^{73}$, eventuali Zone omogenee istituibili pro futuro e Comuni) il ruolo di "deflusso" compensativo dei carichi di lavoro connessi all'esercizio di nuove funzioni $\mathrm{o}$ al diverso esercizio di funzioni già intestate al livello di governo regionale $^{74}$. Nel percorso devolutivo, peraltro, potrebbero utilmente essere coinvolti tutti i soggetti funzionali ${ }^{75}$ rispetto ai quali, in precedenza, si denunciavano le esigenze di raccordo tecnico finora disattese: Università (statali e non), Camere di Commercio (CCIAA), Istituti di Ricovero e Cura a Carattere Scientifico (IRCCS) ed Istituti Clinici Scientifici (ICS), ma anche altri enti pubblici quali Enti di gestione delle aree protette, Parchi regionali (e non), Consorzi di bonifica e di bacino imbrifero montano (BIM), musei etc.

Da quanto descritto deriva logicamente che una seria riflessione intorno all'attuazione dell'art. 116, comma 3, Cost. non dovrebbe prescindere, in ogni caso, da una contestuale ristrutturazione del governo locale, sia sul fronte istituzionale, sia su quello della declinazione delle competenze. La composizione multilivello delle autonomie infraregionali, particolarmente spiccata in Lombardia, dovrebbe essere oggetto di una normativa regionale di razionalizzazione in grado di costituire la piattaforma disciplinare sulla quale incardinare l'intero percorso che prelude all'assunzione di nuovi compiti amministrativi a titolo dell'asimmetria.

Anche in assenza di tale ultimo approdo, peraltro, alla luce della struttura del governo locale lombardo e della sua complessità, una simile prospettiva sembra non più rinviabile.

\footnotetext{
${ }^{73}$ Così sarebbe, ad es., per le Unioni di Comuni. Occorre poi rimarcare che le Comunità montane rientrerebbero formalmente in tale categoria pur accedendo, sovente, ad una dimensione di area vasta, territorialmente più ampia.

74 Il metodo devolutivo e le sue implicazioni non sarebbero poi molto diversi da quanto già proposto in U. РототSCHNIG, La delega di funzioni amministrative regionali agli enti locali, cit..

75 Di questo avviso è anche M. CAMmelli, Flessibilità, autonomia, decentramento amministrativo: il regionalismo oltre l'art.116.3 Cost., cit..
} 
Una corretta riorganizzazione della geografia istituzionale e funzionale del tessuto delle autonomie costituisce un caso emblematico di gioco non a somma zero, nel quale vincono tutti i giocatori - vale a dire tutti gli attori istituzionali, di tutti i livelli di governo - e la sommatoria tra costi e benefici ha valore comunque positivo. Non tutte le riforme presentano tali caratteristiche, anzi, spesso possono condurre anche ad un saldo ampiamente negativo, a causa dei costi di transazione intrinsecamente legati alla riorganizzazione stessa; solamente una riorganizzazione incentrata sulla ricognizione e successiva ponderata riallocazione delle funzioni amministrative - purché correlata a risorse certe e predefinibili per ciascun livello di governo - può costituire invece un esperimento di sicuro successo.

Come occorre muoversi, dunque?

L'intervento del legislatore regionale, che offra alla Regione una normativa unitaria e coerente, capace di compendiare sia i profili istituzionali e funzionali, sia quelli organizzativi ed economico-finanziari, non è più procrastinabile. Una Regione come la Lombardia può spendere molta della propria credibilità sul versante nazionale ed europeo mediante una modernizzazione concreta del proprio governo locale e dei paradigmi di esercizio delle funzioni dei soggetti che operano sul territorio regionale rendendo questa Regione il cuore pulsante delle Alpi.

Le realtà territoriali in difficoltà, siano esse montane o di pianuraancorché apparentemente periferiche rispetto al Capoluogo - possono provocare un effetto di trascinamento all'in giù dell'intero sistema regionale, il quale al contrario ha bisogno di una normativa compatta e coerente, che elimini le distorsioni oggi esistenti ed assicuri il fluido incedere dell'esercizio delle funzioni amministrative.

Nel merito, ciò sottende un consenso sulle seguenti opzioni ${ }^{76}$ :

i. un rinnovato protagonismo regionale - sia da parte del legislatore che del governo regionali - informato alla promozione della differenziazione in tutte le sue possibili forme, che proietti la Regione

76 Un catalogo esaustivo delle ulteriori proposte sperimentabili è presente nelle ricerche promosse e pubblicate dal Dipartimento di Scienze Politiche e Sociali dell'Università di Pavia dal 2018 ad oggi, già citate supra, nt. precedenti. 
in una dimensione di maggiore proattività nel coordinamento dei territori, svincolandola dai ritardi dello Stato;

ii. l'accoglimento di soluzioni di merito informate al miglioramento della governance inter-provinciale (su scala regionale, con regia della Regione) e sub-provinciale (tra i soggetti istituzionali e funzionali operanti in ciascuna Provincia) entrambe propedeutiche a costruire cerniere di raccordo inter-istituzionale flessibili e leggere, nelle quali favorire la formazione del consenso tra amministrazioni ed il coordinamento amministrativo;

iii. il rafforzamento del ruolo delle cc.dd. "Città medie", sicché tali Comuni ben potrebbero esercitare funzioni ulteriori ${ }^{77} \mathrm{ed}$ assolvere ad un ruolo ancora più centrale sia nelle dinamiche territoriali, sia nell'erogazione di servizi pubblici, rispetto a quanto non valga per molti "Comuni polvere" che faticano ad adempiere ai compiti amministrativi loro attribuiti;

iv. la sperimentazione del senso e del ruolo delle Zone omogenee, esercitando la competenza regionale di cui all'art. 33 TUEL in materia di determinazione degli ambiti ottimali per l'esercizio delle funzioni in forma associata, senza creare nuovi livelli di governo ma solo un livello organizzativo ${ }^{78}$ mediano tra la dimensione sovracomunale e quella intercomunale, che sia tracciato sugli stessi confini amministrativi di altri azzonamenti già esistenti ${ }^{79}$;

v. la promozione convinta delle forme associative secondo una logica più rigorosa di elargizione delle risorse, sempre su base volontaristica ed in ragione di progettualità attagliate alle peculiarità territoriali 80.

77 Allo stesso modo, si veda A. VentuRI, Le funzioni ed i servizi di prossimità nella prospettiva del "municipalismo differenziato", in G.C. RICCIARDI, A. VentuRI (a cura), "La riorganizzazione territoriale e funzionale dell'Area vasta", Torino, Giappichelli, 2018.

78 Del resto già U. РототSChNIG, Comuni, comprensori e altre forme associative tra enti locali, in "Rivista trimestrale di Scienza dell'amministrazione", n. 4, 1972, ora in ID., Scritti scelti, cit., era giunto ad affermare che le forme associative non dovessero necessariamente assumere una veste giuridica definita, potendo prescindere dalla costituzione ex novo di un Ente locale apposito in relazione all'ambito territoriale da gestire.

${ }^{79}$ Quali potrebbero essere, ad esempio, gli ambiti di operatività delle ASST o altre circoscrizioni legate alla pianificazione territoriale accolta nel PTR oppure, ancora, altri ambiti concordati a livello provinciale con gli enti di prossimità secondo un approccio bottom$u p$, a valorizzazione delle organizzazioni già esistenti.

${ }^{80} \mathrm{Nel}$ Capo I dedicato alle "Forme collaborative", del titolo IV sugli "Ordinamenti differenziati" (art. 49 ss.) del c.d. "Progetto di Pavia" descritto in AA.vv., Legge generale 
Queste proposte, tuttavia, hanno un senso solo nella sperimentazione del raccordo tra Regione e Province, accompagnato ad una visione sistemica entro la quale maturi una robusta consapevolezza sul fatto che l'efficacia dell'azione amministrativa di queste ultime ed il rilancio delle ontologiche prerogative programmatorie e pianificatorie ad esse storicamente riconosciute ai fini dello sviluppo dei territori ${ }^{81}$ al di là del ridimensionamento funzionale ed organico operato dalla legge 56/2014 e dalla decretazione successiva - appaiono legate "a doppio filo" al destino della Regione stessa. Inoltre, tali proposte possono trovare asilo in una normativa ad hoc che superi l'attuale solo a patto di contemplare in essa tutti i profili sopra rappresentati, che interessano l'intera comunità delle autonomie infraregionali di cui la Regione é soggetto esponenziale 82.

La Regione, infatti, è un reticolo territoriale, un intreccio di azzonamenti che pulsa nel quadro normativo regionale e necessita di un coordinamento autorevole, improntato alla relazionalità. Esso, tuttavia, sottende un impegno regionale attivo e propositivo, espressione di un approccio opposto alla rassegnata attesa di interventi chiarificatori del quadro nazionale, che pure si auspica provengano dal centro. La conquista di maggiori spazi di autonomia prelude a una dialettica con il Governo anche sul fronte del contenzioso in via principale innanzi alla Corte costituzionale, nel caso vi siano buone ragioni ${ }^{83}$ per credere che

sull'amministrazione locale, cit., traspariva la logica di consentire alle amministrazioni la sperimentazione di strumenti duttili e flessibili da sviluppare secondo le caratteristiche della realtà sociale. L'impostazione ivi proposta resta attuale, disvelando potenzialità di ulteriore caratterizzazione su base territoriale.

${ }^{81}$ Soprattutto sul piano dello sviluppo economico, assicurando "[...] la corrispondenza tra natura della funzione e dimensione dell'istituzione" come rammenta G. PASTORI, Intervento programmato, in G. BERTI, G.C. DE MARTIN (a cura), "Il sistema amministrativo dopo la riforma del Titolo V della Costituzione", Roma, Luiss Edizioni, 2002, p. 120.

82 Come insegna G. Berti, Art. 5, in G. Branca (a cura), Commentario alla Costituzione, I, Bologna-Roma, Zanichelli, 1975.

${ }^{83}$ Una buona ragione, ad esempio, è quella della dimensione dell'interesse sotteso alle materie indicate nell'art. 117 Cost.: laddove tale dimensione si palesi effettivamente regionale all'esito della necessaria analisi territoriale, vi sarebbe buon titolo per rivendicare un ruolo più incisivo, anche oltre la rigida ripartizione delle potestà ivi accolta. Rivestono la medesima natura molte questioni di riassetto funzionale afferenti al governo locale, a differenza dei profili di cui all'art. 117, comma 2, lett. $p$ ) in materia di "legislazione elettorale, organi di governo e funzioni fondamentali di Comuni, Province e Città metropolitane", che evidentemente sono ascrivibili all'alveo delle competenze statali. 
valga la pena rivendicare un ruolo e una posizione coerenti con il regionalismo della differenziazione.

Ciascuno, in fondo, anche in un contesto delle competenze di non semplice interpretazione, è artefice del proprio destino.

\begin{abstract}
The paper takes into consideration the current declination of living regionalism, as known so far in our country and how it could be redefined in the light of some emerging dynamics, which enhance the constitutional principle of differentiation. In particular, the paper explores the need for a regional regulation of reorganization of the local government and it frames the potential of the reorganization in the current historicalinstitutional phase crossed by the impulse to-
\end{abstract}

wards further forms and particular conditions of autonomy in favor of the Regions. Finally, the research offers some indications on the reorganization method and on the propositional role that the regional legislator should assume in order to better express the differentiating prerogatives inherent in the powers of the Regions, on the assumption that the Regions themselves are exponential entities of the different levels of local government representing a complex system characterized by autonomy. 